951

A MODEL FOR THE DELIVERY OF INTEGRATED OCCUPATIONAL HEALTH SERVICES WITHIN THE SOUTH AFRICAN PUBLIC HEALTH SYSTEM

${ }^{1} \mathrm{M}$ Govender*, ${ }^{2} \mathrm{~S}$ Rajaram. 'Wits Health Consortium, Johannesburg, South Africa; ${ }^{2}$ School of Public Health, University of Witwatersrand, Johannesburg, South Africa

10.1136/oemed-2018-ICOHabstracts.471

Introduction A comprehensive occupational health service (OHS) should ideally be focused on preventive functions and be informed by good practice for the context in which it is delivered. This is challenge for many countries like South Africa that are facing the quadruple burden of disease with limited resources and have to make informed decisions that will ensure optimum use of resources within the health sector. OHSs tend to be neglected to non-existent in many of these settings especially in those countries that struggle with providing basic health services to the general population. A model is presented here that highlights the potential for an OHS at district level within the public health sector delivering an integrated OHS offering basic primary care services and some selected specialist services for the South African workforce.

Methods This is a conceptual model which has been through various stages of development from a simple 'health laboratory' focus to the current consideration regarding its application for the delivery of evidence based, integrated OHS.

Results The model will be visually explicit when presented in a poster format. It will highlight the innovative nature of the model especially in the context of the existing district health service.

Discussion The delivery of OHS in resource constrained settings remain a challenge for the health sector in South Africa. This conceptual model offers an innovative approach with regards to utilising an existing platform to deliver OHS as part of the Primary Health Care re-engineering process shaping current local health services. It will stimulate discussion on opportunities within the South African Public health sector to consider the delivery of occupational health services going forward.

\section{FROM $<10$ MIU/ML TO $>100$ MIU/ML POST ADMINISTRATION OF COMBINED HEPATITIS A\&B VACCINATION TO NON-RESPONDER HEALTHCARE WORKERS IN THE HSE SOUTH EAST}

AM Howard*. CNM3, Occupational Health, HSE South East

\subsection{6/oemed-2018-ICOHabstracts.472}

Introduction In 2008, the Journal of Infectious Diseases published a paper called' Excellent response rate to a double dose of a combined Hepatitis A and B vaccine in previous non responders to Hepatitis B vaccine. In 2013 the National Immunisation guidelines of Ireland introduced into their Hepatitis B schedule 'administration of a double dose of combined Hepatitis $\mathrm{A}$ and $\mathrm{B}$ vaccine can induce a protective antibody response in some previous non responders' In Occupational Health HSE South East we took this research and guidelines on board and developed a protocol on Combined Hepatitis A and $B$ vaccination.

Method We initially looked at all our Exposure Prone Procedure (EPP) category 1 workers who were non responders to Hepatitis B and offered them the vaccine. Most of these workers had primary and secondary Hepatitis B courses. Since then we have now extended this to our category 2 workers those that are non responders to Hepatitis B but are not EPP workers. Along with development of the protocol we added an episode to our Occupational Health computer package and set up a Combined Hepatitis $\mathrm{A}$ and $\mathrm{B}$ episode with recall schedule at 0,1 and 6 months followed by serology check 2 months post vaccination.

Results To date we have 105 healthcare workers on the Combined Hepatitis A and B course.

67 Females

38 Males

Age Profile $-29 \%$ within the $41-50$ age group, $28 \%$ within the $31-40$ age group and 22\% within the 51-60 age group.

60 Healthcare workers have completed the course and of the 60,55 have seroconverted between $>10 \mathrm{mIU} / \mathrm{ml}$ to over $100 \mathrm{mIU} / \mathrm{ml}$ a success rate so far of $91.6 \%$.

Discussion There are still 25 healthcare workers in the middle of the Combined Hepatitis A and B course, when they are all complete we will have a greater picture. It may be worth opening a discussion on whether Combined Hepatitis A and B vaccine should be considered as a secondary course if the healthcare worker is a non responder after a primary course.

\section{A DESCRIPTION OF THE EFFECTIVENESS OF SCREENING OVERSEAS WORKERS FOR LATENT TB}

${ }^{1} S$ Kelly*, ${ }^{2,3} \mathrm{~A}$ Reid, ${ }^{2,3} \mathrm{P}$ Noone. 'Specialist Registrar in Occupational Medicine, Tallaght Hospital, Dublin, Ireland; ${ }^{2}$ Consultant Occupational Physician, Tallaght Hospital and Coombe Women and Infants University Hospital, Dublin, Ireland; ${ }^{3}$ Consultant Occupational Physician, HSE Dubin North East, Ireland

\subsection{6/oemed-2018-ICOHabstracts.473}

Introduction Healthcare workers(HCW) are at increased risk of aquiring Tuberculosis (TB) than the general population. In Ireland a pre-employment screen for $\mathrm{TB}$ is recommended for all clinical staff working with patients or clinical specimens. Those workers arriving from countries with annual TB rates $>40$ per 100000 population are classified as high priority HCW. Screening involves:

- Questionnaire,

- Tuberulin skin test (TST) or Interferon Gamma Release Assay (IGRA) testing, and

- Chest X-ray.

The aim of our study was to estimate the prevalence of latent $\mathrm{TB}$ in these high priority $\mathrm{HCW}$ and their screening outcomes and follow up.

Methods A retrospective study was carried out on all high priority HCW screened for latent TB between January 2014 and December 2016 across two multicentre occupational health services. We recorded numbers screened, results of TST/IGRA testing, outcome of clinical review and follow up attendance.

Results 505 high priority HCW were screened over 3 years. $17 \%(87 / 505)$ tested positive for latent TB. Of the 74 reviewed at clinic to date, $26 \%(19 / 74)$ completed treatment and $74 \%(55 / 74)$ declined treatment or defaulted from follow up. Number Needed to Screen(NNS) was calculated as 333 based on estimates of a $10 \%$ lifetime risk of reactivation and $70 \%$ relative risk reduction after 6 months of treatment with Isoniazid. $^{1}$

Discussion Of the $17 \%$ of high priority HCW who tested positive for Latent $\mathrm{TB}$, an unexpectedly high proportion (75\%) declined treatment or did not attend for follow up. The NNS 